\title{
Dynamics of High-order Fuzzy Cellular Neural Networks with Time-varying Delays
}

\author{
Manchun Tan ${ }^{1}$, Shuping Xu ${ }^{1}, Z_{h o n g ~} \mathbf{L i}^{2}$ \\ ${ }^{1}$ Department of Mathematics, Jinan University, Guangzhou 510632, P.R.China \\ E-mail:tanmc@jnu.edu.cn (M.C.Tan) xushuping521@sina.cn (S.P.Xu) \\ ${ }^{2}$ Faculty of Mathematics and Computer Science, FernUniversitat in Hagen, Hagen 58084, Germany \\ E-mail: zhong.li@FernUni-Hagen.de
}

Received 30 April 2014

Accepted 21 December 2014

\begin{abstract}
In this paper, dynamic behavior of a class of high-order fuzzy cellular neural networks (HFCNNs) with time-varying delays is investigated. Compared with the previous results in the literature, the restrictions are loosed, since we do not assume the boundedness and monotonicity on the activation functions, and the differentiability of time-varying delay functions. Some sufficient conditions are derived for ascertaining the existence, uniqueness and exponential stability of equilibrium point and uniform boundedness of solutions of the HFCNNs. Finally, two examples are given to show the effectiveness of the proposed criteria, which complement some previously known results.
\end{abstract}

Keywords: High-order neural networks; Equilibrium point; Boundedness; Time-varying delays; Exponential stability.

\section{Introduction}

The fuzzy cellular neural networks (FCNNs), which combines fuzzy logic with the traditional CNNs, has been recognized as an appealing and efficient tool in classification, image processing and pattern recognition, since it was introduced by Yang et al. in 1996 $[1,2]$. Analysis of stability has been the focus of scientific community, because it is required that the neural networks be stable in applications. Various techniques, such as M-matrix, algebraic inequality method, and LMI technique, are employed to derive the stability results of CNNs or FCNNs (see [3] [12] and reference therein).

Recently, the high-order neural networks have attracted considerable attention, as high-order neural networks have stronger approximation property, faster convergence rate, greater storage capacity, and higher fault tolerance than lower-order neural networks (see [13]- [32] and reference therein). Many results on dynamic behavior for various high-order neural networks were reported in the literature [13][32]. By using the generalized Halanay inequality and the fixed point theorem, Guo et al. [15] discussed the global exponential stability and existence of periodic solutions for neural networks. In [17][21], by using Lyapunov functional method, Razumikhin method and LMI technique, the authors have studied the stability of HBAM. Employing nonsingular M-matrix and Lyapunov functional method, Xu et al. [18] studied Hopfields NNs without timedelay, and Jiang et al. [23] studied the exponential stability of HFCNNs with time-varying delays. By using Lyapunov stability theory, LMI and free- 
weighting matrix method, Zheng et al. [25] derived some delay-independent and delay-dependent sufficient conditions for a class of high-order Hopfieldtype neural networks with time-varying delays. By employing a Lyapunov-Krasovskii functional and linear matrix inequality, Wang et al. [30] got some criteria of global exponential stability in the mean square for the reaction-diffusion high-order neural networks. However, the boundedness of the activation functions is assumed in the previous results [16]- [28], and the differentiability of time-varying delays is needed in [23] and [27]- [30]. It is obvious that the assumption on the boundedness of activation functions and the differentiability of timevarying delays would make the results not applicable to some important engineering and physical problems. For example, the Lurie-type activation functions are unbounded. To facilitate the design of neural networks, it is worthwhile to continue to investigate the dynamic behavior of system with more general activation functions and time-varying delays [34]- [37].

Motivated by the above discussion, our objective in this paper is to study the dynamics of high-order fuzzy cellular neural networks (HFCNNs). Without assuming the boundedness on the activation functions and the differentiability of time-varying delays, we will give some criteria on existence, uniqueness and exponential stability of equilibrium point, and prove the uniform boundedness of all solutions of this HFCNNs.

\section{Model Description and Preliminaries}

In this paper, we consider the following delayed $\mathrm{H}$ FCNNs:

$$
\begin{aligned}
\frac{d x_{i}(t)}{d t}= & -d_{i} x_{i}(t)+\sum_{j=1}^{n} a_{i j} f_{j}\left(x_{j}\left(t-\tau_{i j}(t)\right)\right)+\sum_{j=1}^{n} \sum_{l=1}^{n} b_{i j l} g_{j}\left(x_{j}\left(t-\tau_{i j}(t)\right)\right) g_{l}\left(x_{l}\left(t-\tau_{i j}(t)\right)\right) \\
& +\bigwedge_{j=1}^{n} \alpha_{i j} f_{j}\left(x_{j}\left(t-\tau_{i j}(t)\right)\right)+\bigvee_{j=1}^{n} \beta_{i j} f_{j}\left(x_{j}\left(t-\tau_{i j}(t)\right)\right) \\
& +\bigwedge_{j=1}^{n}\left(\bigwedge_{l=1}^{n} \gamma_{i j l} g_{j}\left(x_{j}\left(t-\tau_{i j}(t)\right)\right) g_{l}\left(x_{l}\left(t-\tau_{i j}(t)\right)\right)\right) \\
& +\bigvee_{j=1}^{n}\left(\bigvee_{l=1}^{n} \sigma_{i j l} g_{j}\left(x_{j}\left(t-\tau_{i j}(t)\right)\right) g_{l}\left(x_{l}\left(t-\tau_{i j}(t)\right)\right)\right) \\
& +\bigwedge_{j=1}^{n} T_{i j} \mu_{j}+\bigvee_{j=1}^{n} H_{i j} \mu_{j}+I_{i},
\end{aligned}
$$

with the initial condition

$$
x_{i}(t)=\varphi_{i}(t), \quad-h \leqslant t \leqslant 0,
$$

where $i, j, l=1,2, \ldots, n . x_{i}(t)$ denotes the potential (or voltage) of the cell $i$ at time $t ; d_{i}$ is positive constant, which denotes the rate with which the $i$-th cell resets its potential to the resting state when isolated from the other cells and inputs; $\tau_{i j}(t)$ represents the transmission time-varying delay, which satisfies $0 \leqslant \tau_{i j}(t) \leqslant h ; a_{i j}$ and $b_{i j l}$ are the first-order and second-order connection weights of neural network, respectively; $\alpha_{i j}, \beta_{i j}, T_{i j}$ and $H_{i j}$ are elements of the first-order fuzzy feedback MIN template, first-order fuzzy feedback MAX template, first-order fuzzy feed-forward MIN template and first-order fuzzy feed-forward MAX template, respectively; $\gamma_{i j l}$ and $\sigma_{i j l}$ are elements of the second-order fuzzy feedback MIN template, second-order fuzzy feedback MAX template, 
respectively; $\bigwedge$ and $\bigvee$ denote the fuzzy AND and fuzzy OR operations, respectively; $\mu_{i}$ and $I_{i}$ denote input and bias of the $i$-th neurons, respectively; $f_{i}, g_{i}$ are the activation functions.

For convenience, we introduce the following notations. $R^{+}=[0,+\infty) . C[X, Y]$ is a continuous mapping set from the topological space $X$ to the topological space $Y$. For $x(t): R^{+} \rightarrow R^{n}$, we define $\|x(t)\|=\max _{1 \leqslant i \leqslant n}\left\{q_{i}\left|x_{i}(t)\right|\right\}$, and for the initial value $\varphi \in C$ we define $\|\varphi\|_{h}=\sup _{-h \leqslant s \leqslant 0}\{\|\varphi(s)\|\}$, where $\|\varphi(s)\|=\max _{1 \leqslant i \leqslant n}\left\{q_{i}\left|\varphi_{i}(s)\right|\right\}$.

$$
\begin{gathered}
M=\max _{1 \leqslant i \leqslant n} \sum_{j=1}^{n} \frac{\left(\left|a_{i j}\right|+\left|\alpha_{i j}\right|+\left|\beta_{i j}\right|\right) q_{i} q_{j}^{-1} L_{j}}{d_{i}}, \\
L=\max _{1 \leqslant i \leqslant n} \sum_{j=1}^{n} \sum_{l=1}^{n} \frac{2\left(\left|b_{i j l}\right|+\left|\gamma_{i j l}\right|+\left|\sigma_{i j l}\right|\right) q_{i} q_{j}^{-1} q_{l}^{-1} K_{j} K_{l}}{d_{i}}, \\
P=\max _{1 \leqslant i \leqslant n} \frac{q_{i}}{d_{i}}\left[\sum_{j=1}^{n}\left|a_{i j} f_{j}(0)\right|+\bigwedge_{j=1}^{n}\left|\alpha_{i j} f_{j}(0)\right|+\bigvee_{j=1}^{n}\left|\beta_{i j} f_{j}(0)\right|+\bigwedge_{j=1}^{n}\left|T_{i j} \mu_{j}\right|+\bigvee_{j=1}^{n}\left|H_{i j} \mu_{j}\right|+\left|I_{i}\right|\right], \\
D_{1}=\frac{1-M-\sqrt{(1-M)^{2}-4 L P}}{2 L}, \\
D_{2}=\frac{1-M+\sqrt{(1-M)^{2}-4 L P}}{2 L} .
\end{gathered}
$$

For system (1), we make the following assumptions:

(H1) There are some positive constants $L_{i}, K_{i}$ such that

$$
\left|f_{i}(\mu)-f_{i}(v)\right| \leqslant L_{i}|\mu-v|, \quad\left|g_{i}(\mu)-g_{i}(v)\right| \leqslant K_{i}|\mu-v|,
$$

$\mu, v \in R, g_{i}(0)=0, i=1,2, \ldots, n$.

(H2) There exist constants $q_{i}>0, i=1,2, \ldots, n$, such that $M<1$ and

$$
(M-1)^{2}-4 L P>0 .
$$

To obtain our main results, we need the following lemma.

Lemma 1 [2] Suppose $x=\left(x_{1}, x_{2}, \ldots x_{n}\right)$ and $y=\left(y_{1}, y_{2}, \ldots y_{n}\right)$ are two states of system (1), then we have

$$
\begin{aligned}
& \left|\bigwedge_{j=1}^{n} \alpha_{i j} f_{i}\left(x_{j}\right)-\bigwedge_{j=1}^{n} \alpha_{i j} f_{i}\left(y_{j}\right)\right| \leqslant \sum_{j=1}^{n}\left|\alpha_{i j}\right|\left|f_{i}\left(x_{j}\right)-f_{i}\left(y_{j}\right)\right| \\
& \left|\bigvee_{j=1}^{n} \alpha_{i j} f_{i}\left(x_{j}\right)-\bigvee_{j=1}^{n} \alpha_{i j} f_{i}\left(y_{j}\right)\right| \leqslant \sum_{j=1}^{n}\left|\alpha_{i j}\right|\left|f_{i}\left(x_{j}\right)-f_{i}\left(y_{j}\right)\right|
\end{aligned}
$$




\section{Main results}

Theorem 1 Under the assumptions (H1)-(H2), system (1) has a unique equilibrium point $x^{*}$ in the region $S=\left\{\varphi \in C \mid \quad\|\varphi\|_{h} \leqslant d\right\}$, where $d \in\left[D_{1}, D_{2}\right)$ is a constant.

Proof: If $x^{*}=\left(x_{1}{ }^{*}, x_{1}{ }^{*}, \cdots, x_{n}{ }^{*}\right)$ is an equilibrium of system (1), $x^{*}$ satisfies the following equation:

$$
\begin{aligned}
x_{i}^{*}= & \frac{1}{d_{i}}\left[\sum_{j=1}^{n} a_{i j} f_{j}\left(x_{j}^{*}\right)+\bigwedge_{j=1}^{n} \alpha_{i j} f_{j}\left(x_{j}^{*}\right)+\bigvee_{j=1}^{n} \beta_{i j} f_{j}\left(x_{j}^{*}\right)+\sum_{j=1}^{n} \sum_{l=1}^{n} b_{i j l} g_{j}\left(x_{j}^{*}\right) g_{l}\left(x_{l}^{*}\right)\right. \\
& +\bigwedge_{j=1}^{n}\left(\bigwedge_{l=1}^{n} \gamma_{i j l} g_{j}\left(x_{j}^{*}\right) g_{l}\left(x_{l}^{*}\right)\right)+\bigvee_{j=1}^{n}\left(\bigvee_{l=1}^{n} \sigma_{i j l} g_{j}\left(x_{j}^{*}\right) g_{l}\left(x_{l}^{*}\right)\right) \\
& \left.+\bigwedge_{j=1}^{n} T_{i j} \mu_{j}+\bigvee_{j=1}^{n} H_{i j} \mu_{j}+I_{i}\right], \quad i=1,2, \ldots, n .
\end{aligned}
$$

Let $\Omega=\{x \in C \mid\|x\| \leqslant d\}$. It is obvious that $\Omega \subset S$ holds. Consider a mapping $F: \Omega \rightarrow R^{n}$ defined by

$$
\begin{aligned}
F_{i}(x)= & \frac{1}{d_{i}}\left[\sum_{j=1}^{n} a_{i j} f_{j}\left(x_{j}\right)+\bigwedge_{j=1}^{n} \alpha_{i j} f_{j}\left(x_{j}\right)+\bigvee_{j=1}^{n} \beta_{i j} f_{j}\left(x_{j}\right)+\sum_{j=1}^{n} \sum_{l=1}^{n} b_{i j l} g_{j}\left(x_{j}\right) g_{l}\left(x_{l}\right)\right. \\
& +\bigwedge_{j=1}^{n}\left(\bigwedge_{l=1}^{n} \gamma_{i j l} g_{j}\left(x_{j}\right) g_{l}\left(x_{l}\right)\right)+\bigvee_{j=1}^{n}\left(\bigvee_{l=1}^{n} \sigma_{i j l} g_{j}\left(x_{j}\right) g_{l}\left(x_{l}\right)\right) \\
& \left.+\bigwedge_{j=1}^{n} T_{i j} \mu_{j}+\bigvee_{j=1}^{n} H_{i j} \mu_{j}+I_{i}\right], \quad i=1,2, \ldots, n
\end{aligned}
$$

where $F(x)=\left(F_{1}(x), F_{2}(x), \ldots F_{n}(x)\right)^{T}$.

Without loss of generality, we assume that $P \neq 0$. From the assumption (H2), we know the equation $L d^{2}+$ $(M-1) d+P=0$ has two solutions $D_{1}$ and $D_{2}$. For any $d \in\left[D_{1}, D_{2}\right)$, we have

$$
L d^{2}+(M-1) d+P \leqslant 0 \quad \text { and } \quad M+L d<1 .
$$

From assumption (H1), we obtain $\left|f_{j}\left(x_{j}\right)-f_{j}(0)\right| \leqslant L_{j}\left|x_{j}\right|$ and

$$
\begin{aligned}
& \sum_{j=1}^{n} \sum_{l=1}^{n} b_{i j l} g_{j}\left(x_{j}\right) g_{l}\left(x_{l}\right)+\bigwedge_{j=1}^{n}\left(\bigwedge_{l=1}^{n} \gamma_{i j l} g_{j}\left(x_{j}\right) g_{l}\left(x_{l}\right)\right)+\bigvee_{j=1}^{n}\left(\bigvee_{l=1}^{n} \sigma_{i j l} g_{j}\left(x_{j}\right) g_{l}\left(x_{l}\right)\right) \\
\leqslant & \sum_{j=1}^{n} \sum_{l=1}^{n}\left(\left|b_{i j l}\right|+\left|\gamma_{i j l}\right|+\left|\sigma_{i j l}\right|\right) K_{j} K_{l}\left|x_{j}\right|\left|x_{l}\right|
\end{aligned}
$$

For any $x \in \Omega$, and $i=1,2, \ldots, n$, we can get 


$$
\begin{aligned}
& q_{i}\left|F_{i}(x)\right|=\frac{q_{i}}{d_{i}} \mid \sum_{j=1}^{n} a_{i j} f_{j}\left(x_{j}\right)+\bigwedge_{j=1}^{n} \alpha_{i j} f_{j}\left(x_{j}\right)+\bigvee_{j=1}^{n} \beta_{i j} f_{j}\left(x_{j}\right)+\sum_{j=1}^{n} \sum_{l=1}^{n} b_{i j l} g_{j}\left(x_{j}\right) g_{l}\left(x_{l}\right) \\
& +\bigwedge_{j=1}^{n}\left(\bigwedge_{l=1}^{n} \gamma_{i j l} g_{j}\left(x_{j}\right) g_{l}\left(x_{l}\right)\right)+\bigvee_{j=1}^{n}\left(\bigvee_{l=1}^{n} \sigma_{i j l} g_{j}\left(x_{j}\right) g_{l}\left(x_{l}\right)\right)+\bigwedge_{j=1}^{n} T_{i j} \mu_{j}+\bigvee_{j=1}^{n} H_{i j} \mu_{j}+I_{i} \mid \\
& =\frac{q_{i}}{d_{i}} \mid \sum_{j=1}^{n} a_{i j} f_{j}\left(x_{j}\right)-\sum_{j=1}^{n} a_{i j} f_{j}(0)+\bigwedge_{j=1}^{n} \alpha_{i j} f_{j}\left(x_{j}\right)-\bigwedge_{j=1}^{n} \alpha_{i j} f_{j}(0)+\bigvee_{j=1}^{n} \beta_{i j} f_{j}\left(x_{j}\right)-\bigvee_{j=1}^{n} \beta_{i j} f_{j}(0) \\
& +\sum_{j=1}^{n} \sum_{l=1}^{n} b_{i j l} g_{j}\left(x_{j}\right) g_{l}\left(x_{l}\right)+\bigwedge_{j=1}^{n}\left(\bigwedge_{l=1}^{n} \gamma_{i j l} g_{j}\left(x_{j}\right) g_{l}\left(x_{l}\right)\right)+\bigvee_{j=1}^{n}\left(\bigvee_{l=1}^{n} \sigma_{i j l} g_{j}\left(x_{j}\right) g_{l}\left(x_{l}\right)\right)+\sum_{j=1}^{n} a_{i j} f_{j}(0) \\
& +\bigwedge_{j=1}^{n} \alpha_{i j} f_{j}(0)+\bigvee_{j=1}^{n} \beta_{i j} f_{j}(0)+\bigwedge_{j=1}^{n} T_{i j} \mu_{j}+\bigvee_{j=1}^{n} H_{i j} \mu_{j}+I_{i} \mid \\
& \leqslant \frac{q_{i}}{d_{i}}\left[\sum_{j=1}^{n}\left|\left(a_{i j}|+| \alpha_{i j}|+| \beta_{i j} \mid\right) L_{j}\right| x_{j}\left|+\sum_{j=1}^{n} \sum_{l=1}^{n} 2\left(\left|b_{i j l}\right|+\left|\gamma_{i j l}\right|+\left|\sigma_{i j l}\right|\right) K_{j} K_{l}\right| x_{j}|| x_{l} \mid\right. \\
& \left.+\sum_{j=1}^{n}\left|a_{i j} f_{j}(0)\right|+\bigwedge_{j=1}^{n}\left|\alpha_{i j} f_{j}(0)\right|+\bigvee_{j=1}^{n}\left|\beta_{i j} f_{j}(0)\right|+\bigwedge_{j=1}^{n}\left|T_{i j} \mu_{j}\right|+\bigvee_{j=1}^{n}\left|H_{i j} \mu_{j}\right|+\left|I_{i}\right|\right] . \\
& =\sum_{j=1}^{n} \frac{\left(\left|a_{i j}\right|+\left|\alpha_{i j}\right|+\left|\beta_{i j}\right|\right) q_{i} q_{j}^{-1} L_{j}}{d_{i}} q_{j}\left|x_{j}\right| \\
& +\sum_{j=1}^{n} \sum_{l=1}^{n} \frac{2\left(\left|b_{i j l}\right|+\left|\gamma_{i j l}\right|+\left|\sigma_{i j l}\right|\right) q_{i} q_{j}^{-1} q_{l}^{-1} K_{j} K_{l}}{d_{i}} q_{j}\left|x_{j}\right| q_{l}\left|x_{l}\right| \\
& +\frac{q_{i}}{d_{i}}\left[\sum_{j=1}^{n}\left|a_{i j} f_{j}(0)\right|+\bigwedge_{j=1}^{n}\left|\alpha_{i j} f_{j}(0)\right| \bigvee_{j=1}^{n}\left|\beta_{i j} f_{j}(0)\right|+\bigwedge_{j=1}^{n}\left|T_{i j} \mu_{j}\right|+\bigvee_{j=1}^{n}\left|H_{i j} \mu_{j}\right|+\left|I_{i}\right|\right] \\
& \leqslant \sum_{j=1}^{n} \frac{\left(\left|a_{i j}\right|+\left|\alpha_{i j}\right|+\left|\beta_{i j}\right|\right) q_{i} q_{j}^{-1} L_{j}}{d_{j}}\|x\| \\
& +\sum_{j=1}^{n} \sum_{l=1}^{n} \frac{2\left(\left|b_{i j l}\right|+\left|\gamma_{i j l}\right|+\left|\sigma_{i j l}\right|\right) q_{i} q_{j}^{-1} q_{l}^{-1} K_{j} K_{l}}{d_{i}}\|x\|^{2} \\
& +\frac{q_{i}}{d_{i}}\left[\sum_{j=1}^{n}\left|a_{i j} f_{j}(0)\right|+\bigwedge_{j=1}^{n}\left|\alpha_{i j} f_{j}(0)\right| \bigvee_{j=1}^{n}\left|\beta_{i j} f_{j}(0)\right|+\bigwedge_{j=1}^{n}\left|T_{i j} \mu_{j}\right|+\bigvee_{j=1}^{n}\left|H_{i j} \mu_{j}\right|+\left|I_{i}\right|\right]
\end{aligned}
$$




$$
\begin{aligned}
\leqslant & \max _{1 \leqslant i \leqslant n} \sum_{j=1}^{n} \frac{\left(\left|a_{i j}\right|+\left|\alpha_{i j}\right|+\left|\beta_{i j}\right|\right) q_{i} q_{j}^{-1} L_{j}}{d_{j}} d \\
& +\max _{1 \leqslant i \leqslant n} \sum_{j=1}^{n} \sum_{l=1}^{n} \frac{2\left(\left|b_{i j l}\right|+\left|\gamma_{i j l}\right|+\left|\sigma_{i j l}\right|\right) q_{i} q_{j}^{-1} q_{l}^{-1} K_{j} K_{l}}{d_{i}} d^{2} \\
& +\max _{1 \leqslant i \leqslant n} \frac{q_{i}}{d_{i}}\left[\sum_{j=1}^{n}\left|a_{i j} f_{j}(0)\right|+\bigwedge_{j=1}^{n}\left|\alpha_{i j} f_{j}(0)\right| \bigvee_{j=1}^{n}\left|\beta_{i j} f_{j}(0)\right|+\bigwedge_{j=1}^{n}\left|T_{i j} \mu_{j}\right|+\bigvee_{j=1}^{n}\left|H_{i j} \mu_{j}\right|+\left|I_{i}\right|\right] \\
= & M d+L d^{2}+P \\
\leqslant & d .
\end{aligned}
$$

Accordingly, we have $\|f(x)\|=\max _{1 \leqslant i \leqslant n}\left\{q_{i}\left|F_{i}\left(x_{i}\right)\right|\right\} \leqslant d$, which implies $F$ maps the set $\Omega$ into itself. By the Brower's fixed point theorem [33], we get $F\left(x^{*}\right)=x^{*}, x^{*} \in \Omega$, i.e., the system (1) has a solution.

Assume that $x=\left(x_{1}, x_{2}, \ldots x_{n}\right)$ and $y=\left(y_{1}, y_{2}, \ldots y_{n}\right)$ are two solutions of system (1), then

$$
\begin{aligned}
x_{i}= & \frac{1}{d_{i}}\left[\sum_{j=1}^{n} a_{i j} f_{j}\left(x_{j}\right)+\bigwedge_{j=1}^{n} \alpha_{i j} f_{j}\left(x_{j}\right)+\bigvee_{j=1}^{n} \beta_{i j} f_{j}\left(x_{j}\right)+\sum_{j=1}^{n} \sum_{l=1}^{n} b_{i j l} g_{j}\left(x_{j}\right) g_{l}\left(x_{l}\right)\right. \\
& \left.+\bigwedge_{j=1}^{n}\left(\bigwedge_{l=1}^{n} \gamma_{i j l} g_{j}\left(x_{j}\right) g_{l}\left(x_{l}\right)\right)+\bigvee_{j=1}^{n}\left(\bigvee_{l=1}^{n} \sigma_{i j l} g_{j}\left(x_{j}\right) g_{l}\left(x_{l}\right)\right)+\bigwedge_{j=1}^{n} T_{i j} \mu_{j}+\bigvee_{j=1}^{n} H_{i j} \mu_{j}+I_{i}\right], \\
y_{i}= & \frac{1}{d_{i}}\left[\sum_{j=1}^{n} a_{i j} f_{j}\left(y_{j}\right)+\bigwedge_{j=1}^{n} \alpha_{i j} f_{j}\left(y_{j}\right)+\bigvee_{j=1}^{n} \beta_{i j} f_{j}\left(y_{j}\right)+\sum_{j=1}^{n} \sum_{l=1}^{n} b_{i j l} g_{j}\left(y_{j}\right) g_{l}\left(y_{l}\right)\right. \\
& \left.+\bigwedge_{j=1}^{n}\left(\bigwedge_{l=1}^{n} \gamma_{i j l} g_{j}\left(y_{j}\right) g_{l}\left(y_{l}\right)\right)+\bigvee_{j=1}^{n}\left(\bigvee_{l=1}^{n} \sigma_{i j l} g_{j}\left(y_{j}\right) g_{l}\left(y_{l}\right)\right)+\bigwedge_{j=1}^{n} T_{i j} \mu_{j}+\bigvee_{j=1}^{n} H_{i j} \mu_{j}+I_{i}\right] .
\end{aligned}
$$

Thus,

$$
\begin{aligned}
\left|x_{i}-y_{i}\right|= & \frac{1}{d_{i}} \mid \sum_{j=1}^{n} a_{i j} f_{j}\left(x_{j}\right)-\sum_{j=1}^{n} a_{i j} f_{j}\left(y_{j}\right)+\bigwedge_{j=1}^{n} \alpha_{i j} f_{j}\left(x_{j}\right)-\bigwedge_{j=1}^{n} \alpha_{i j} f_{j}\left(y_{j}\right) \\
& +\bigvee_{j=1}^{n} \beta_{i j} f_{j}\left(x_{j}\right)-\bigvee_{j=1}^{n} \beta_{i j} f_{j}\left(y_{j}\right)+\sum_{j=1}^{n} \sum_{l=1}^{n} b_{i j l} g_{j}\left(x_{j}\right) g_{l}\left(x_{l}\right)-\sum_{j=1}^{n} \sum_{l=1}^{n} b_{i j l} g_{j}\left(y_{j}\right) g_{l}\left(y_{l}\right) \\
& +\bigwedge_{j=1}^{n}\left(\bigwedge_{l=1}^{n} \gamma_{i j l} g_{j}\left(x_{j}\right) g_{l}\left(x_{l}\right)\right)-\bigwedge_{j=1}^{n}\left(\bigwedge_{l=1}^{n} \gamma_{i j l} g_{j}\left(y_{j}\right) g_{l}\left(y_{l}\right)\right) \\
& +\bigvee_{j=1}^{n}\left(\bigvee_{l=1}^{n} \sigma_{i j l} g_{j}\left(x_{j}\right) g_{l}\left(x_{l}\right)\right)-\bigvee_{j=1}^{n}\left(\bigvee_{l=1}^{n} \sigma_{i j l} g_{j}\left(y_{j}\right) g_{l}\left(y_{l}\right)\right) \mid
\end{aligned}
$$

$i=1,2, \ldots, n$. From (9), we have 


$$
\begin{aligned}
q_{i}\left|x_{i}-y_{i}\right| \leqslant & \frac{q_{i}}{d_{i}}\left[\sum_{j=1}^{n}\left(\left|a_{i j}\right|+\left|\alpha_{i j}\right|+\left|\beta_{i j}\right|\right) L_{j}\left|x_{j}-y_{j}\right|\right. \\
& \left.+\sum_{j=1}^{n} \sum_{l=1}^{n}\left(\left|b_{i j l}\right|+\left|\gamma_{i j l}\right|+\left|\sigma_{i j l}\right|\right)\left|g_{j}\left(x_{j}\right) g_{l}\left(x_{l}\right)-g_{j}\left(y_{j}\right) g_{l}\left(y_{l}\right)\right|\right] \\
\leqslant & \frac{q_{i}}{d_{i}}\left[\sum_{j=1}^{n}\left(\left|a_{i j}\right|+\left|\alpha_{i j}\right|+\left|\beta_{i j}\right|\right) L_{j}\left|x_{j}-y_{j}\right|\right. \\
& \left.+\sum_{j=1}^{n} \sum_{l=1}^{n}\left(\left|b_{i j l}\right|+\left|\gamma_{i j l}\right|+\left|\sigma_{i j l}\right|\right) K_{j} K_{l}\left(\left|x_{j}\right|\left|x_{l}-y_{l}\right|+\left|y_{l}\right|\left|x_{j}-y_{j}\right|\right)\right] \\
\leqslant & \frac{q_{i}}{d_{i}}\left[\sum_{j=1}^{n}\left(\left|a_{i j}\right|+\left|\alpha_{i j}\right|+\left|\beta_{i j}\right|\right) L_{j} q_{j}^{-1}\|x-y\|\right. \\
& \left.+\sum_{j=1}^{n} \sum_{l=1}^{n}\left(\left|b_{i j l}\right|+\left|\gamma_{i j l}\right|+\left|\sigma_{i j l}\right|\right) K_{j} K_{l} q_{j}^{-1} q_{l}^{-1}(\|x\|\|x-y\|+\|y\|\|x-y\|)\right] \\
\leqslant & M\|x-y\|+L d\|x-y\| \\
= & (M+L d)\|x-y\|, \quad i=1,2, \ldots, n .
\end{aligned}
$$

Thus $\|x-y\|=\max _{1 \leqslant i \leqslant n}\left\{q_{i}\left|x_{i}-y_{i}\right|\right\} \leqslant(M+L d)\|x-y\|$. If $\|x-y\| \neq 0$, we have $M+L d \geqslant 1$, which contradicts $M+L d<1$. Hence $x=y$. The proof is complete.

Theorem 2 Under the assumptions (H1)-(H2), all the solutions of system (1) are uniformly bounded in the region $S=\left\{\varphi \in C \mid\|\varphi\|_{h} \leqslant d\right\}$, where $d \in\left[D_{1}, D_{2}\right)$ is a constant.

Proof: We will show that $\|x(t)\| \leqslant d, \quad d \in\left[D_{1}, D_{2}\right)$, for $t \geqslant 0$, where $x(t)$ is a solution of the system (1) with the initial $\varphi \in S$.

To prove $\|x(t)\| \leqslant d, \quad d \in\left[D_{1}, D_{2}\right)$, we first show that, for any $d \in\left[D_{1}, D_{2}\right)$, there exists $\beta>1$, such that $\|x(t)\|<\beta d, \quad \beta d \in\left(D_{1}, D_{2}\right), t \geqslant 0$.

If $\|x(t)\|<\beta d, \quad \beta d \in\left(D_{1}, D_{2}\right), t \geqslant 0$ is not true, there must be some $t_{1}>0$ such that

$$
\left\|x\left(t_{1}\right)\right\|=\beta d, \quad D^{-}\left(\left\|x\left(t_{1}\right)\right\|\right) \geqslant 0
$$

and

$$
\|x(t)\|<\beta d, \quad 0 \leqslant t<t_{1}
$$

hold.

By the definition of the vector norm, there exists $i \in\{1,2, \ldots, n\}$, so that

$$
\left\|x\left(t_{1}\right)\right\|=q_{i}\left|x_{i}\left(t_{1}\right)\right|=\beta d, \quad D^{-}\left(\left\|x\left(t_{1}\right)\right\|\right) \geqslant 0
$$

and

$$
q_{j}\left|x_{j}\left(t_{1}\right)\right|<\beta d, \quad 0 \leqslant t<t_{1}, \quad j=1,2, \ldots, n .
$$


From the system (1), we have

$$
\begin{aligned}
D^{-}\left(q_{i}\left|x_{i}(t)\right|\right)= & q_{i} \dot{x}_{i}(t) \operatorname{sgn}\left(x_{i}(t)\right) \\
= & {\left[-q_{i} d_{i} x_{i}(t)+q_{i} \sum_{j=1}^{n} a_{i j} f\left(x_{j}\left(t-\tau_{i j}(t)\right)\right)+q_{i} \bigwedge_{j=1}^{n} \alpha_{i j} f_{j}\left(x_{j}\left(t-\tau_{i j}(t)\right)\right)\right.} \\
& +q_{i} \bigvee_{j=1}^{n} \beta_{i j} f_{j}\left(x_{j}\left(t-\tau_{i j}(t)\right)\right)+q_{i} \sum_{j=1}^{n} \sum_{l=1}^{n} b_{i j l} g_{j}\left(x_{j}\left(t-\tau_{i j}(t)\right)\right) g_{l}\left(x_{l}\left(t-\tau_{i j}(t)\right)\right) \\
& +q_{i} \bigwedge_{j=1}^{n}\left(\bigwedge_{l=1}^{n} \gamma_{i j l} g_{j}\left(x_{j}\left(t-\tau_{i j}(t)\right)\right) g_{l}\left(x_{l}\left(t-\tau_{i j}(t)\right)\right)\right) \\
& +q_{i} \bigvee_{j=1}^{n}\left(\bigvee_{l=1}^{n} \sigma_{i j l} g_{j}\left(x_{j}\left(t-\tau_{i j}(t)\right)\right) g_{l}\left(x_{l}\left(t-\tau_{i j}(t)\right)\right)\right) \\
& \left.+q_{i} \bigwedge_{j=1}^{n} T_{i j} \mu_{j}+q_{i} \bigvee_{j=1}^{n} H_{i j} \mu_{j}+q_{i} I_{i}\right] \operatorname{sgn}\left(x_{i}(t)\right) .
\end{aligned}
$$

From (13), we get

$$
\begin{aligned}
D^{-}\left(q_{i}\left|x_{i}\left(t_{1}\right)\right|\right) \leqslant & -q_{i} d_{i}\left|x_{i}\left(t_{1}\right)\right|+q_{i} \sum_{j=1}^{n}\left(\left|a_{i j}\right|+\left|\alpha_{i j}\right|+\left|\beta_{i j}\right|\right) L_{j}\left|x_{j}\left(t_{1}-\tau_{i j}\left(t_{1}\right)\right)\right| \\
& +q_{i} \sum_{j=1}^{n} \sum_{l=1}^{n} 2\left(\left|b_{i j l}\right|+\left|\gamma_{i j l}\right|+\left|\sigma_{i j l}\right|\right) K_{j} K_{l}\left|x_{j}\left(t_{1}-\tau_{i j}\left(t_{1}\right)\right)\right|\left|x_{l}\left(t_{1}-\tau_{i j}\left(t_{1}\right)\right)\right| \\
& +q_{i}\left[\sum_{j=1}^{n}\left|a_{i j} f_{j}(0)\right|+\bigwedge_{j=1}^{n}\left|\alpha_{i j} f_{j}(0)\right|+\bigvee_{j=1}^{n}\left|\beta_{i j} f_{j}(0)\right|\right. \\
& \left.+\bigwedge_{j=1}^{n}\left|T_{i j} \mu_{j}\right|+\bigvee_{j=1}^{n}\left|H_{i j} \mu_{j}\right|+\left|I_{i}\right|\right] \\
\leqslant & d_{i}\left\{-\beta d+\max _{1 \leqslant i \leqslant n} \sum_{j=1}^{n} \frac{\left(\left|a_{i j}\right|+\left|\alpha_{i j}\right|+\left|\beta_{i j}\right|\right) q_{i} q_{j}^{-1} L_{j}}{d_{i}} \beta d\right. \\
& +\max _{1 \leqslant i \leqslant n} \sum_{j=1}^{n} \sum_{l=1}^{n} \frac{2\left(\left|b_{i j l}\right|+\left|\gamma_{i j l}\right|+\left|\sigma_{i j l}\right|\right) q_{i} q_{j}^{-1} q_{l}^{-1} K_{j} K_{l}}{d_{i}}(\beta d)^{2} \\
& +\max _{1 \leqslant i \leqslant n} \frac{q_{i}}{d_{i}}\left[\sum_{j=1}^{n}\left|a_{i j} f_{j}(0)\right|+\bigwedge_{j=1}^{n}\left|\alpha_{i j} f_{j}(0)\right|+\bigvee_{j=1}^{n}\left|\beta_{i j} f_{j}(0)\right|\right. \\
& \left.\left.+\bigwedge_{j=1}^{n}\left|T_{i j} \mu_{j}\right|+\bigvee_{j=1}^{n}\left|H_{i j} \mu_{j}\right|+\left|I_{i}\right|\right]\right\} \\
= & d_{i}\left[-\beta d+M(\beta d)+L(\beta d)^{2}+P\right] \\
= & d_{i}\left[L(\beta d)^{2}+(M-1)(\beta d)+P\right]<0,
\end{aligned}
$$

which contradicts the inequality (9). So $\|x(t)\|<\beta d, \quad \beta d \in\left(D_{1}, D_{2}\right)$ holds. Let $\beta \rightarrow 1$, we have $\|x(t)\|<$ $d, \quad d \in\left[D_{1}, D_{2}\right)$. The proof is complete. 
Theorem 3 Under the assumptions (H1)-(H2), the equilibrium of the system (1) is exponentially stable in the region $S=\left\{\varphi \in C \mid \quad\|\varphi\|_{h} \leqslant d\right\}$, where $d \in\left[D_{1}, D_{2}\right)$ is a constant.

Proof: Suppose $x^{*}$ is the equilibrium of system (1). Let $y(t)=x(t)-x^{*}$, then system (1) can be transformed into the following system:

$$
\begin{aligned}
\frac{d y_{i}}{d t}= & -d_{i} y_{i}(t)+\sum_{j=1}^{n} a_{i j} f_{j}\left(x_{j}\right)-\sum_{j=1}^{n} a_{i j} f_{j}\left(x_{j}^{*}\right) \\
& +\bigwedge_{j=1}^{n} \alpha_{i j} f_{j}\left(x_{j}\right)-\bigwedge_{j=1}^{n} \alpha_{i j} f_{j}\left(x_{j}^{*}\right)+\bigvee_{j=1}^{n} \beta_{i j} f_{j}\left(x_{j}\right)-\bigvee_{j=1}^{n} \beta_{i j} f_{j}\left(x_{j}^{*}\right) \\
& +\sum_{j=1}^{n} \sum_{l=1}^{n} b_{i j l} g_{j}\left(x_{j}\right) g_{l}\left(x_{l}\right)-\sum_{j=1}^{n} \sum_{l=1}^{n} b_{i j l} g_{j}\left(x_{j}^{*}\right) g_{l}\left(x_{l}^{*}\right) \\
& +\bigwedge_{j=1}^{n}\left(\bigwedge_{l=1}^{n} \gamma_{i j l} g_{j}\left(x_{j}\right) g_{l}\left(x_{l}\right)\right)-\bigwedge_{j=1}^{n}\left(\bigwedge_{l=1}^{n} \gamma_{i j l} g_{j}\left(x_{j}^{*}\right) g_{l}\left(x_{l}^{*}\right)\right) \\
& +\bigvee_{j=1}^{n}\left(\bigvee_{l=1}^{n} \sigma_{i j l} g_{j}\left(x_{j}\right) g_{l}\left(x_{l}\right)\right)-\bigvee_{j=1}^{n}\left(\bigvee_{l=1}^{n} \sigma_{i j l} g_{j}\left(x_{j}^{*}\right) g_{l}\left(x_{l}^{*}\right)\right)
\end{aligned}
$$

with the initial condition

$$
y_{i}(t)=\phi_{i}(t), \quad-h \leqslant t \leqslant 0
$$

where $i, j, l=1,2, \ldots, n, \phi_{i}(t)=\varphi_{i}(t)-x_{i}^{*}$. Obviously, to prove equilibrium $x^{*}$ of the the system (1) is exponentially stable, we will only consider the exponential stability of the equilibrium point $O$ of the system (15).

From the inequality $M+L d<1$, there exists $\lambda, 0<\lambda \ll \min _{1 \leqslant i \leqslant n}\left\{d_{i}\right\}$, such that $\eta+\frac{\lambda}{\min _{1 \leqslant i \leqslant n}\left\{d_{i}\right\}}<1$, where

$$
\eta=\max _{1 \leqslant i \leqslant n} \sum_{j=1}^{n} \frac{\left(\left|a_{i j}\right|+\left|\alpha_{i j}\right|+\left|\beta_{i j}\right|\right) q_{i} q_{j}^{-1} L_{j} e^{\lambda h}}{d_{i}}+\max _{1 \leqslant i \leqslant n} \sum_{j=1}^{n} \sum_{l=1}^{n} \frac{2\left(\left|b_{i j l}\right|+\left|\gamma_{i j l}\right|+\left|\sigma_{i j l}\right|\right) q_{i} q_{j}^{-1} q_{l}^{-1} K_{j} K_{l} d e^{\lambda h}}{d_{i}} .
$$

Let $V(t)=\|y(t)\| e^{\lambda t}$, for any $\varphi \in S$, we will show that

$$
V(t) \leqslant \frac{1}{1-\eta}\|\phi\|_{h}, \quad \forall t \geqslant 0
$$

where $\phi=\varphi-x^{*}$.

To prove (16), we first show that for any given $\xi>1$,

$$
V(t) \leqslant \frac{\xi}{1-\eta}\|\phi\|_{h}
$$

holds for all $t \geqslant 0$.

Similar to the discussion of Theorem 2, if the inequality (17) is not true, there must be exists $t_{2} \geqslant 0$, such that

$$
V\left(t_{2}\right)=\frac{\xi}{1-\eta}\|\phi\|_{h}, \quad D^{-}\left(V\left(t_{2}\right)\right) \geqslant 0
$$


and

$$
V\left(t_{2}\right)<\frac{\xi}{1-\eta}\|\phi\|_{h}, \quad 0 \leqslant t<t_{2} .
$$

By the definition of the vector norm, there exists $i \in\{1,2, \ldots, n\}$, such that

$$
V\left(t_{2}\right)=\left\|y\left(t_{2}\right)\right\| e^{\lambda t_{2}}=q_{i}\left|y_{i}\left(t_{2}\right)\right| e^{\lambda t_{2}}=\frac{\xi}{1-\eta}\|\phi\|_{h}, \quad D^{-}\left(V\left(t_{2}\right)\right) \geqslant 0
$$

Obviously,

$$
\begin{aligned}
D^{-}(V(t))= & D^{-}\left(q_{i}\left|y_{i}(t)\right| e^{\lambda t}\right) \\
= & \lambda q_{i}\left|y_{i}(t)\right| e^{\lambda t}+q_{i} \dot{y}_{i}(t) e^{\lambda t} \operatorname{sgn}\left(y_{i}(t)\right) \\
= & \lambda q_{i}\left|y_{i}(t)\right| e^{\lambda t}+q_{i} e^{\lambda t} \operatorname{sgn}\left(y_{i}(t)\right)\left[-d_{i} y_{i}(t)+\sum_{j=1}^{n} a_{i j} f_{j}\left(x_{j}\left(t-\tau_{i j}(t)\right)\right)\right. \\
& -\sum_{j=1}^{n} a_{i j} f_{j}\left(x_{j}^{*}\right)+\bigwedge_{j=1}^{n} \alpha_{i j} f_{j}\left(x_{j}\left(t-\tau_{i j}(t)\right)\right)-\bigwedge_{j=1}^{n} \alpha_{i j} f_{j}\left(x_{j}^{*}\right) \\
& +\bigvee_{j=1}^{n} \beta_{i j} f_{j}\left(x_{j}\left(t-\tau_{i j}(t)\right)\right)-\bigvee_{j=1}^{n} \beta_{i j} f_{j}\left(x_{j}^{*}\right) \\
& +\sum_{j=1}^{n} \sum_{l=1}^{n} b_{i j l} g_{j}\left(x_{j}\left(t-\tau_{i j}(t)\right)\right) g_{l}\left(x_{l}\left(t-\tau_{i j}(t)\right)\right)-\sum_{j=1}^{n} \sum_{l=1}^{n} b_{i j l} g_{j}\left(x_{j}^{*}\right) g_{l}\left(x_{l}^{*}\right) \\
& +\bigwedge_{j=1}^{n}\left(\bigwedge_{l=1}^{n} \gamma_{i j l} g_{j}\left(x_{j}\left(t-\tau_{i j}(t)\right)\right) g_{l}\left(x_{l}\left(t-\tau_{i j}(t)\right)\right)\right)-\bigwedge_{j=1}^{n}\left(\bigwedge_{l=1}^{n} \gamma_{i j l} g_{j}\left(x_{j}^{*}\right) g_{l}\left(x_{l}^{*}\right)\right) \\
& \left.+\bigvee_{j=1}^{n}\left(\bigvee_{l=1}^{n} \sigma_{i j l} g_{j}\left(x_{j}\left(t-\tau_{i j}(t)\right)\right) g_{l}\left(x_{l}\left(t-\tau_{i j}(t)\right)\right)\right)-\bigvee_{j=1}^{n}\left(\bigvee_{l=1}^{n} \sigma_{i j l} g_{j}\left(x_{j}^{*}\right) g_{l}\left(x_{l}^{*}\right)\right)\right]
\end{aligned}
$$

We obtain

$$
\begin{aligned}
D^{-}\left(V\left(t_{2}\right)\right) \leqslant & \lambda q_{i}\left|y_{i}\left(t_{2}\right)\right| e^{\lambda t_{2}} \\
& +q_{i} e^{\lambda t_{2}}\left[-d_{i}\left|y_{i}\left(t_{2}\right)\right|+\sum_{j=1}^{n}\left(\left|a_{i j}\right|+\left|\alpha_{i j}\right|+\left|\beta_{i j}\right|\right)\left|F_{j}\left(y_{j}\left(t_{2}-\tau_{i j}\left(t_{2}\right)\right)\right)\right|\right. \\
& \left.+\sum_{j=1}^{n} \sum_{l=1}^{n}\left(\left|b_{i j l}\right|+\left|\gamma_{i j l}\right|+\left|\sigma_{i j l}\right|\right)\left|G_{j}\left(y_{j}\left(t_{2}-\tau_{i j}\left(t_{2}\right)\right)\right) G_{l}\left(y_{l}\left(t_{2}-\tau_{i j}\left(t_{2}\right)\right)\right)\right|\right],
\end{aligned}
$$

where

$$
\begin{aligned}
& F_{j}\left(y_{j}\left(t_{2}-\tau_{i j}\left(t_{2}\right)\right)\right)=f_{j}\left(x_{j}\left(t_{2}-\tau_{i j}\left(t_{2}\right)\right)\right)-f_{j}\left(x_{j}^{*}\right), \\
& G_{j}\left(y_{j}\left(t_{2}-\tau_{i j}\left(t_{2}\right)\right)\right) G_{l}\left(y_{l}\left(t_{2}-\tau_{i j}\left(t_{2}\right)\right)\right)=g_{j}\left(x_{j}\left(t_{2}-\tau_{i j}\left(t_{2}\right)\right)\right) g_{l}\left(x_{l}\left(t_{2}-\tau_{i j}\left(t_{2}\right)\right)\right)-g_{j}\left(x_{j}^{*}\right) g_{l}\left(x_{l}^{*}\right) .
\end{aligned}
$$

From the assumption (H1), we have

$$
\left|F_{j}\left(y_{j}\left(t_{2}-\tau_{i j}\left(t_{2}\right)\right)\right)\right| \leqslant L_{j}\left|y_{j}\left(t_{2}-\tau_{i j}\left(t_{2}\right)\right)\right|
$$


and

$$
\begin{aligned}
& \left|G_{j}\left(y_{j}\left(t_{2}-\tau_{i j}\left(t_{2}\right)\right)\right) G_{l}\left(y_{l}\left(t_{2}-\tau_{i j}\left(t_{2}\right)\right)\right)\right| \\
= & \mid g_{j}\left(x_{j}\left(t_{2}-\tau_{i j}\left(t_{2}\right)\right)\right) g_{l}\left(x_{l}\left(t_{2}-\tau_{i j}\left(t_{2}\right)\right)\right)-g_{j}\left(x_{j}\left(t_{2}-\tau_{i j}\left(t_{2}\right)\right)\right) g_{l}\left(x_{l}^{*}\right) \\
& +g_{j}\left(x_{j}\left(t_{2}-\tau_{i j}\left(t_{2}\right)\right)\right) g_{l}\left(x_{l}^{*}\right)-g_{j}\left(x_{j}^{*}\right) g_{l}\left(x_{l}^{*}\right) \mid \\
\leqslant & K_{j}\left|x_{j}\left(t_{2}-\tau_{i j}\left(t_{2}\right)\right)\right| K_{l}\left|x_{l}\left(t_{2}-\tau_{i j}\left(t_{2}\right)\right)-x_{l}^{*}\right|+K_{l}\left|x_{l}^{*}\right| K_{j}\left|x_{j}\left(t_{2}-\tau_{i j}\left(t_{2}\right)\right)-x_{j}^{*}\right| \\
= & K_{j} K_{l}\left|y_{j}\left(t_{2}-\tau_{i j}\left(t_{2}\right)\right)+x_{l}^{*}\right|\left|y_{l}\left(t_{2}-\tau_{i j}\left(t_{2}\right)\right)\right|+K_{j} K_{l}\left|x_{l}^{*}\right|\left|y_{j}\left(t_{2}-\tau_{i j}\left(t_{2}\right)\right)\right| .
\end{aligned}
$$

Thus,

$$
\begin{aligned}
D^{-}\left(V\left(t_{2}\right)\right) \leqslant & \lambda q_{i}\left|y_{i}\left(t_{2}\right)\right| e^{\lambda t_{2}}+q_{i} e^{\lambda t_{2}}\left[-d_{i}\left|y_{i}\left(t_{2}\right)\right|+\sum_{j=1}^{n}\left(\left|a_{i j}\right|+\left|\alpha_{i j}\right|+\left|\beta_{i j}\right|\right) L_{j}\left|y_{j}\left(t_{2}-\tau_{i j}\left(t_{2}\right)\right)\right|\right. \\
& +\sum_{j=1}^{n} \sum_{l=1}^{n}\left(\left|b_{i j l}\right|+\left|\gamma_{i j l}\right|+\left|\sigma_{i j l}\right|\right) K_{j} K_{l} \\
& \left.\times\left(\left|y_{j}\left(t_{2}-\tau_{i j}\left(t_{2}\right)\right)+x_{l}^{*}\right|\left|y_{l}\left(t_{2}-\tau_{i j}\left(t_{2}\right)\right)\right|+\left|x_{l}^{*}\right|\left|y_{j}\left(t_{2}-\tau_{i j}\left(t_{2}\right)\right)\right|\right)\right] \\
= & \lambda q_{i}\left|y_{i}\left(t_{2}\right)\right| e^{\lambda t_{2}}-d_{i} q_{i}\left|y_{i}\left(t_{2}\right)\right| e^{\lambda t_{2}}+\sum_{j=1}^{n}\left(\left|a_{i j}\right|+\left|\alpha_{i j}\right|+\left|\beta_{i j}\right|\right) L_{j} q_{i} q_{j}^{-1} q_{j}\left|y_{j}\left(t_{2}-\tau_{i j}\left(t_{2}\right)\right)\right| e^{\lambda t_{2}} \\
& +\sum_{j=1}^{n} \sum_{l=1}^{n}\left(\left|b_{i j l}\right|+\left|\gamma_{i j l}\right|+\left|\sigma_{i j l}\right|\right) K_{j} K_{l} q_{i} q_{j}^{-1} q_{l}^{-1} q_{j} q_{l} \\
& \times\left[\left|y_{j}\left(t_{2}-\tau_{i j}\left(t_{2}\right)\right)+x_{l}^{*}\right|\left|y_{l}\left(t_{2}-\tau_{i j}\left(t_{2}\right)\right)\right|+\left|x_{l}^{*}\right|\left|y_{j}\left(t_{2}-\tau_{i j}\left(t_{2}\right)\right)\right|\right] e^{\lambda t_{2}} \\
\leqslant & \lambda \frac{\xi}{1-\eta}\|\Phi\|_{h}-d_{i} \frac{\xi}{1-\eta}\|\Phi\|_{h}+\sum_{j=1}^{n}\left(\left|a_{i j}\right|+\left|\alpha_{i j}\right|+\left|\beta_{i j}\right|\right) L_{j} q_{i} q_{j}^{-1} \frac{\xi}{1-\eta}\|\Phi\|_{h} e^{\lambda h} \\
& +\sum_{j=1}^{n} \sum_{l=1}^{n} 2\left(\left|b_{i j l}\right|+\left|\gamma_{i j l}\right|+\left|\sigma_{i j l}\right|\right) K_{j} K_{l} q_{i} q_{j}^{-1} q_{l}^{-1} \frac{\xi}{1-\eta} d\|\Phi\|_{h} e^{\lambda h} \\
\leqslant & \xi\|\Phi\|_{h}\left[\frac{\lambda}{1-\eta}+\frac{d_{i}}{1-\eta}\left(-1+\max _{1 \leqslant i \leqslant n} \sum_{j=1}^{n} \frac{\left(\left|a_{i j}\right|+\left|\alpha_{i j}\right|+\left|\beta_{i j}\right|\right) q_{i} q_{j}^{-1} L_{j} e^{\lambda h}}{d_{i}}\right.\right. \\
& \left.\left.+\max _{1 \leqslant i \leqslant n} \sum_{j=1}^{n} \sum_{l=1}^{n} \frac{2\left(\left|b_{i j l}\right|+\left|\gamma_{i j l}\right|+\left|\sigma_{i j l}\right|\right) q_{i} q_{j}^{-1} q_{l}^{-1} K_{j} K_{l} d e^{\lambda h}}{d_{i}}\right)\right] \\
= & \xi\|\Phi\|_{h} \times\left[\frac{\lambda}{1-\eta}+\frac{d_{i}}{1-\eta}(\eta-1)\right] \\
= & \xi\|\Phi\|_{h} \times\left(\frac{\lambda}{1-\eta}-d_{i}\right)<0,
\end{aligned}
$$

which contradicts $D^{-}\left(V\left(t_{2}\right)\right) \geqslant 0$. So $V(t)=\|y(t)\| e^{\lambda t}<\frac{\xi}{1-\eta}\|\Phi\|_{h}$ holds. Let $\xi \rightarrow 1$, then (16) holds. That is

$$
\left\|x(t)-x^{*}\right\|=\|y(t)\| \leqslant \frac{1}{1-\eta}\|\Phi\|_{h} e^{-\lambda t}=\frac{1}{1-\eta}\left\|\varphi-x^{*}\right\| e^{-\lambda t} .
$$

The proof is complete. 
Remark 1. The HFCNNs in [23] is a special case of system (1) with $f_{i}=g_{i}$. In [23] the activation functions $f_{i}$ and $g_{i}$ in system (1) are assumed to be bounded, and these restrictions are not needed in this paper.

Remark 2. Let $\alpha_{i j}=0, \beta_{i j}=0, \gamma_{i j l}=0, \sigma_{i j l}=$ $0, T_{i j}=0, H_{i j}=0$ in the system (1), then it degenerates into special cases of the systems which were studied in [20] [22] [24] [25] [27] [28] [31]. Even specially, if $\tau_{i j}(t)=0$ holds, system (1) becomes the model in [18]. However, the boundedness of activation functions is necessary in these mentioned work$\mathrm{s}$, the derivative of the time delays is required to be less than one in [27], and the monotonicity of activation functions must be satisfied in [31], while these limitations are removed in our results.

\section{Illustrative Example}

Example 1. Consider the 2-dimension HFCNNs with time delays. For system (1), we take activation functions $f_{i}(x)=\frac{1}{2}(|1+x|+|1-x|), g_{i}(x)=\frac{2}{5}(\mid x+$ $1|-| x-1 \mid)(i=1,2)$. Obviously, $f_{i}(x), g_{i}(x)$ satisfies Hypotheses (H1), where $L_{i}=1, K_{i}=0.8(i=$ $1,2)$, we take $q_{1}=q_{2}=1, \tau_{i j}(t)=2+|\sin (t)|(i, j=$ $1,2), I_{1}=1, I_{2}=-1, \mu_{1}=\mu_{2}=1$,

$$
\begin{gathered}
D=\operatorname{diag}\left\{d_{1}, d_{2}\right\}=\left[\begin{array}{cc}
8 & 0 \\
0 & 9
\end{array}\right], \\
A=\left\{a_{i j}\right\}_{2 \times 2}=\left[\begin{array}{cc}
\frac{1}{5} & \frac{1}{10} \\
-\frac{1}{10} & \frac{1}{5}
\end{array}\right], \\
\alpha=\left\{\alpha_{i j}\right\}_{2 \times 2}=\left[\begin{array}{cc}
\frac{1}{10} & -\frac{1}{10} \\
\frac{1}{5} & \frac{1}{10}
\end{array}\right], \\
\beta=\left\{\beta_{i j}\right\}_{2 \times 2}=\left[\begin{array}{cc}
\frac{1}{10} & \frac{1}{5} \\
-\frac{1}{5} & \frac{1}{10}
\end{array}\right], \\
B_{1}=\left\{b_{1 j l}\right\}_{2 \times 2}=\left[\begin{array}{cc}
\frac{1}{10} & -\frac{1}{10} \\
\frac{1}{5} & \frac{1}{10}
\end{array}\right], \\
B_{2}=\left\{b_{2 j l}\right\}_{2 \times 2}=\left[\begin{array}{cc}
\frac{1}{10} & \frac{1}{8} \\
\frac{1}{8} & -\frac{1}{10}
\end{array}\right], \\
R_{1}=\left\{\gamma_{1 j l}\right\}_{2 \times 2}=\left[\begin{array}{cc}
-\frac{1}{10} & \frac{1}{8} \\
\frac{1}{5} & -\frac{1}{4}
\end{array}\right],
\end{gathered}
$$

$$
R_{2}=\left\{\gamma_{2 j l}\right\}_{2 \times 2}=\left[\begin{array}{cc}
-\frac{1}{10} & \frac{1}{10} \\
\frac{1}{8} & \frac{1}{10}
\end{array}\right]
$$

$$
Q_{1}=\left\{\sigma_{1 j l}\right\}_{2 \times 2}=\left[\begin{array}{cc}
\frac{1}{10} & \frac{1}{5} \\
\frac{1}{4} & -\frac{1}{8}
\end{array}\right] \text {, }
$$$$
Q_{2}=\left\{\sigma_{2 j l}\right\}_{2 \times 2}=\left[\begin{array}{cc}
\frac{1}{5} & -\frac{1}{4} \\
\frac{1}{10} & -\frac{1}{10}
\end{array}\right]
$$$$
T=\left\{T_{i j}\right\}_{2 \times 2}=\left[\begin{array}{cc}
\frac{1}{10} & -\frac{1}{8} \\
\frac{1}{5} & \frac{1}{8}
\end{array}\right],
$$

$$
H=\left\{H_{i j}\right\}_{2 \times 2}=\left[\begin{array}{cc}
\frac{1}{10} & -\frac{1}{5} \\
\frac{1}{8} & \frac{1}{5}
\end{array}\right] .
$$

By simple calculation, we have $M=0.1<1, L=$ $0.296, P=0.2375$, and $(1-M)^{2}-4 L P>0$ which implies the hypotheses (H1)-(H2) hold. Therefore, it follows from Theorems 1-3 that the system has a unique equilibrium point $x^{*}$ which is exponentially stable in the region $S=\left\{\varphi \in C \mid\|\varphi\|_{h} \leqslant d\right\}$, where $d \in[0.2920,2.7486)$ is a constant.

Since the activation functions are unbounded and the time-delays are not differentiable in Example 1, the results in [23] is not applicable to this system.

Example 2. If $\alpha_{i j}=0, \beta_{i j}=0, \gamma_{i j l}=0, \sigma_{i j l}=$ $0, T_{i j}=0, H_{i j}=0$ in the system (1), and the other parameters are the same as that in Example 1, then the system (1) becomes a Hopfield type network. Let $f_{i}(x)=g_{i}(x)=|x|$ and choose $L_{i}=K_{i}=1$ and $q_{i}=1(i=1,2)$. We get $M=0.0375, L=0.125$ and $P=0.1625$. It is easy to know that the conditions in Theorems 1-3 are satisfied, so the system has a unique exponentially stable equilibrium point. However the results that are mentioned in Remark 2 cannot be used to ascertain the stability of this neural networks. 


\section{Conclusions}

In this paper, the dynamic behavior of HFCNNs model with time-varying delays has been investigated. By using Brouwer's fixed point theorem and employing inequality technique, some sufficient criteria ensuring the existence, uniqueness and exponential stability of equilibrium point and uniform boundedness of HFCNNs have been derived. Two examples are used to show that our results are new and complement some previously known results, since the activation functions are not necessarily bounded or monotonic, and the time-varying delays are not necessarily differentiable.

\section{Acknowledgments}

The research is supported by grants from the National Natural Science Foundation of China (No.11471083), the Natural Science Foundation of Guangdong Province in China (No.9151001003000005), and the Fundamental Research Funds for the Central Universities (No. 21612443).

\section{References}

1. T. Yang, L. B. Yang, C. W. Wu, L.O. Chua, "Fuzzy cellular neural networks:theory," In : Proceedings of the IEEE International Workshop on cellular Neural Networks and their Applications, pp.181-186 (1996).

2. T. Yang, L. B. Yang, "Global stability of fuzzy cellular neural network,'IEEE Trans Circuits Syst I Fundam Theory Appl, 43, 880-883 (1996).

3. K. Yuan, J. D. Cao, J. M. Deng, "Exponential stability and periodic solutions of fuzzy cellular neural networks with time-varying delays," Neurocomputing , 69, 1619-1627 (2006).

4. M. C. Tan, "Exponential convergence behavior of fuzzy cellular neural networks with distributed delays and time-varying coefficients,"International Journal of Bifurcation and Chaos, 19, 2455-2462 (2009).

5. M. C. Tan, "Global asymptotic stability of fuzzy cellular neural networks with unbounded distributed delays," Neural Process Lett, 31,147-157 (2010).

6. M. C. Tan, "Global exponential stability of delayed fuzzy cellular neural networks," International Journal of Fuzzy Systems, 12,199-207(2010).

7. T. W. Huang, "Exponential stability of delayed fuzzy cellular neural networks with distributed delay,"Physics Letters A, 351, 48-52 (2006).

8. Q. H. Zhang, R. G. Xiang, "Global asymptotic stability of fuzzy cellular neural networks with time-varying delays,"Physics Letters A, 372, 3971-3977 (2008).

9. Q. X. Zhu, X. D. Li, "Exponential and almost sure exponential stability of stochastic fuzzy delayed CohenGrossberg neural networks,"Fuzzy Sets and Systems, 203, 74-94 (2012).

10. R. Chandran, P. Balasubramaniam, "Delay dependen$\mathrm{t}$ exponential stability for fuzzy recurrent neural networks with interval time-varying delay,"Neural Processing Letters, 37, 147-161 (2013).

11. C. D. Zheng, Y. Wang, Z. S. Wang, "Global stability of fuzzy cellular neural networks with mixed delays and leakage delay under impulsive perturbations,"Circuits Systems and Signal Processing, 33, 10671094 (2014).

12. C. D. Zheng, Y. Wang, Z. S. Wang, "Stability analysis of stochastic fuzzy Markovian jumping neural networks with leakage delay under impulsive perturbations," Journal of the Franklin Institute-Engineering and Applied Mathematics, 351, 1728-1755 (2014).

13. A. Dembo, O. Farotimi, T. Kailath, "High-order absolutely stable neural networks. IEEE Trans,"Circuits Syst, 38, 57-65 (1991).

14. H. B. Gu, H. J. Jiang, Z. D. Teng, "On the dynamics in high-order cellular neural networks with time-varying delays," Differ Equ Dyn Syst, 19, 119-132 (2011).

15. H. B. Gu, H. J. Jiang, Z. D. Teng, "Stability and periodicity in high-order neural networks with impulsive effects," Nonlinear Analysis: Theory, Methods \& Applications, 68, 3186-3200 (2008).

16. B. J. Xu, X. Z. Liu, X. X. Liao, " Global asymptotic stability of high-order Hopfield type neural networks with time delays," Comput. Math. Appl. 45, 17291737 (2003).

17. J. D. Cao, J. L. Liang, J. Lam, "Exponential stability of high-order bidirectional associative memory neural networks with time delays,"Physica D, 199, 425-436 (2004).

18. B. J. Xu, X. Z. Liu, X. X. Liao, "Global exponential stability of high order Hopfield type neural networks,"Applied Mathematics and Computation, 174, 98116 (2006).

19. D. W. C. Ho, J. L. Liang, J. Lam, "Global exponential stability of impulsive high-order BAM neural networks with time-varying delays,"Neural Networks, 19, 1581-1590 (2006).

20. B.Y. Zhang, S.Y. Xu, Y.M. Li, Y.M. Chu, "On global exponential stability of high-order neural networks with time-varying delays," Physics Letters A, 366, 69-78 (2007).

21. C. J. Li, C. D. Li, X. F. Liao, T. W. Huang, "Impulsive effects on stability of high-order BAM neural net- 
works with time delays,"Neurocomputing, 74, 15411550 (2011)

22. F. Qiu, B. T. Cui, W. Wu, "Global exponential stability of high order recurrent neural network with timevarying delays,"Applied Mathematical Modelling, 33, 198-210 (2009).

23. H. J. Jiang, B. J. Guo, Z. D. Teng, "Exponential stability of high-Order fuzzy cellular neural networks with time-varying delays," Lecture Notes in Computer Science, 5551, 413-422 (2009).

24. J. L. Qiu, "Dynamics of high-order Hopfield neural networks with time delays,"Neurocomputing, 73, 820-826 (2010).

25. C. D. Zheng, H. G. Zhang, Z. S. Wang, "Novel exponential stability criteria of high-order neural networks with time-varying delays,"IEEE Trans Syst Man Cybern B Cybern, 41, 486-496 (2011).

26. Y. F. Wang, C. G. Lu, G. R. Ji, L. S. Wang, "Global exponential stability of high-order Hopfield-type neural networks with S-type distributed time delays,"Communications in Nonlinear Science and $\mathrm{Nu}$ merical Simulation, 16, 3319-3325 (2011).

27. F. L. Ren, J. D. Cao, "LMI-based criteria for stability of high-order neural networks with time-varying delay,"Nonlinear Analysis: Real World Applications, 7, 967-979 (2006).

28. X. Y. Lou, B. T. Cui, "Novel global stability criteria for high-order Hopfield-type neural networks with time-varying delays,"J Math Anal Appl, 330, 144-158 (2007).

29. Z. Huang, Q. G. Yang, "Exponential stability of impulsive high-order cellular neural networks with timevarying delays,"Nonlinear Analysis: Real World Ap- plications, 11, 592-600 (2010).

30. Y. F. Wang, P. Lin, L. S. Wang, "Exponential stability of reaction-diffusion high-order Markovian jump Hopfield neural networks with time-varying delays,"Nonlinear Analysis: Real World Applications, 13, 1353-1361 (2012).

31. B. J. Xua, Q. Wang, X. X. Liao, "Stability analysis of high-order Hopfield type neural networks with uncertainty," Neurocomputing, 71, 508-512 (2008).

32. A. C. Zhang, J. L. Qiu, J. H. She, "Existence and global exponential stability of periodic solution for highorder discrete-time BAM neural networks,"Neural Networks, 50 98-109 (2014).

33. J. K. Hale, S.M.V. Lunel, "Introduction to Functional Differential Equations," Springer-Verlag, New York, (1991).

34. H. Zhang, W. T. Wang, B. Xiao, "Exponential convergence for high-order recurrent neural networks with a class of general activation functions,"Applied Mathematical Modelling, 35, 123-129 (2011).

35. A. L. Wu, Z. G. Zeng, C. J. Fu, W. W. Shen, “ Global exponential stability in Lagrange sense for periodic neural networks with various activation functions," Neurocomputing, 74, 831-837 (2011).

36. O. Faydasicok, S. Arik, “ An analysis of stability of uncertain neural networks with multiple time delays," Journal of the Franklin Institute, 350, 18081826 (2013).

37. J. G. Jian, B. X. Wang, “ Stability analysis in Lagrange sense for a class of BAM neural networks of neutral type with multiple time-varying delays," Neurocomputing, 149, 930-939 (2015). 